\title{
The risk of ventricular arrhythmias in a Dutch CRT population: CRT-defibrillator versus CRT-pacemaker
}

\author{
I.A.H. ter Horst ${ }^{1} \cdot$ J. van 't Sant ${ }^{2} \cdot$ S.C. Wijers ${ }^{1,3}$. \\ M.A. $\operatorname{Vos}^{3} \cdot$ M.J. Cramer ${ }^{1} \cdot$ M. Meine ${ }^{1}$
}

Published online: 21 January 2016

(C) The Author(s) 2016. This article is published with open access at Springerlink.com

\begin{abstract}
Background Patients eligible for cardiac resynchronisation therapy (CRT) have an indication for primary prophylactic implantable cardioverter defibrillator (ICD) therapy. However, response to CRT might influence processes involved in arrhythmogenesis and therefore change the necessity of ICD therapy in certain patients.

Method In 202 CRT-defibrillator patients, the association between baseline variables, 6-month echocardiographic outcome (volume response: left ventricular end-systolic volume decrease $<\geq 15 \%$ and left ventricular ejection fraction (LVEF) $\leq>35 \%$ ) and the risk of first appropriate ICD therapy was analysed retrospectively.

Results Fifty (25\%) patients received appropriate ICD therapy during a median follow-up of 37 (23-52) months. At baseline ischaemic cardiomyopathy (hazard ratio (HR) 2.0, $p=0.019$ ) and a B-type natriuretic peptide level $>163 \mathrm{pmol} / \mathrm{l}$ (HR $3.8, p<0.001$ ) were significantly associated with the risk of appropriate ICD therapy. After 6 months, $105(52 \%)$ patients showed volume response and $51(25 \%)$ reached an LVEF $>35 \%$. Three $(6 \%)$ patients with an LVEF $>35 \%$ received appropriate ICD therapy following echocardiography at \pm 6 months compared with 43 patients
\end{abstract}

I.A.H. ter Horst

i.a.h.terhorst@umcutrecht.nl

1 Department of Cardiology, University Medical Center Utrecht, Heidelberglaan 100, 3508 GA PO Box 85500, Utrecht, The Netherlands

2 Department of Cardiology, St. Antonius Hospital Nieuwegein, Nieuwegein, The Netherlands

3 Department of Medical Physiology, University Medical Center Utrecht, Utrecht, The Netherlands
(29\%) with an LVEF $\leq 35 \%$ ( $p=0.001)$. LVEF post-CRT was more strongly associated to the risk of ventricular arrhythmias than volume response (LVEF $>35 \%$, HR 0.23 , $p=0.020$ ).

Conclusion Assessing the necessity of an ICD in patients eligible for CRT remains a challenge. Six months post-CRT an LVEF $>35 \%$ identified patients at low risk of ventricular arrhythmias. LVEF might be used at the time of generator replacement to identify patients suitable for downgrading to a CRT-pacemaker.

Keywords Cardiac resynchronisation therapy ·

Ventricular arrhythmias · Appropriate ICD

\section{Introduction}

The majority of patients eligible for cardiac resynchronisation therapy (CRT) also have an indication for primary prophylactic implantable cardioverter defibrillator (ICD) therapy based on their depressed left ventricular ejection fraction (LVEF $\leq 35 \%$ ) [1]. Although LVEF is the strongest predictor of ventricular arrhythmias in patients without a history of ventricular arrhythmias, its value in patients eligible for CRT is questionable. CRT aims to improve LVEF and induces reverse remodelling of the left ventricle. Both reverse remodelling and overcrossing a certain threshold in LVEF post-CRT have previously been linked to a decreased risk of ventricular arrhythmias, but inconclusively [2-11]. As a CRT-defibrillator (CRT-D) device is much more expensive than a CRT-pacemaker (CRT-P) and the additional ICD induces the risk of inappropriate ICD therapy, it is clinically relevant to identify those patients at low risk of ventricular arrhythmias prior to CRT-D implant or at the time of CRT-D generator replacement [12]. 


\title{
Advertisement placed here.
}

\author{
CSS Bohn

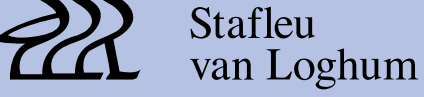 \\ Springer Media
}

Houten 2015 


\title{
Advertisement placed here.
}

\author{
CSS Bohn

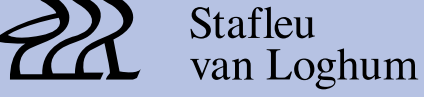 \\ Springer Media
}

Houten 2015 
Therefore, in the current study we aimed to (I) identify baseline predictors of appropriate ICD therapy after the start of CRT; (II) assess whether we can confirm the link between LVEF improvement and reverse remodelling and a lower risk of ventricular arrhythmias; and (III) which echocardiographic outcome parameter, volume response or absolute LVEF, is the best predictor of ventricular arrhythmias and therefore should be used for risk stratification at time of replacement.

\section{Method}

\section{Study design and population}

This retrospective study analysed data from all patients who received either a de novo CRT-D or a CRT-D upgrade at the University Medical Center of Utrecht (UMCU) in the period 2005-2011. Only patients receiving an ICD for primary prevention were included in the analysis, thereby excluding upgrade patients with prior appropriate ICD therapy. Indication for CRT was based on European Society of Cardiology (ESC) guidelines, [13] evidence-based medicine and inclusion criteria of CRT studies [14]. Patients were included if echocardiographic data were available and analysable 1 month before and 6 months after CRT implantation.

\section{Clinical, laboratory and electrocardiography data}

Baseline clinical and laboratory data were collected by reviewing hospital records.

Electrocardiographic characteristics as bundle branch block and QRS duration were analysed on pre-implantation electrocardiograms (ECG) using criteria set by the American Heart Association to define bundle branch block morphology [15].

\section{Echocardiography data}

Echocardiographic studies were performed using a Vivid 7 (General Electric, Milwaukee, USA). Patients were imaged in the left lateral decubitus position. At baseline, volumes, LVEF, and interventricular mechanical delay (IVMD) were assessed. Six months after device implantation, volumes and LVEF were evaluated again during biventricular pacing. LV volumes and LVEF were measured according to Simpson's biplane method. Measurements were performed three times and averaged. IVMD was defined as the timing difference between opening of the aortic valve and the pulmonary valve. Doppler flows over the pulmonary and aortic valve were recorded and time from $\mathrm{Q}$ to onset of flow was assessed for both valves.

\section{Echocardiographic outcome}

Echocardiographic outcome groups were based on a relative decrease in LV end-systolic volume of $<\geq 15 \%$ (non-responders vs. volume responders respectively), and overcrossing the threshold in absolute LVEF for primary prophylactic ICD therapy of $35 \%$ after 6 months of CRT [16].

\section{Endpoint}

The endpoint was the occurrence of first appropriate ICD therapy during follow-up, including both anti-tachycardia pacing and shock, with notification of time until first appropriate ICD discharge. Episodes of ICD therapy were analysed by experienced cardiologists. Follow-up started directly after implant to assess baseline predictors of appropriate ICD therapy. Ventricular arrhythmias episodes occurring before the 6-month echocardiography were excluded to assess the best echocardiographic outcome predictor of ICD therapy. Patient-specific follow-up ended with the last ICD check-up and the end of all follow-up was the end of December 2013. ICD device settings were collected from the electronic patient files.

\section{Statistical analysis}

IBM SPSS statistics 20.0 (Chicago, Illinois) was used for statistical analysis. Continuous variables were tested for normal distribution by Kolmogorov-Smirnov tests. Normally distributed variables are presented as mean with standard deviation and compared using Student's T-test. In case of skewed distribution, variables are presented as median with interquartile range (IQR) and compared using the Mann-Whitney U test. Categorical variables are presented as frequencies with percentages and compared using Pearson's chi-square test.

To assess baseline predictors of appropriate ICD therapy univariate Cox regression analysis was performed. LVEF at baseline, QRS duration and IVMD were dichotomised based on cut-offs that were previously used in the literature: $20 \%, 150$ and $40 \mathrm{~ms}$, respectively [17]. Other continuous variables were dichotomised by using the optimal cut-off in the receiver operating characteristic (ROC) curves of their association with first appropriate ICD therapy. Correlation between variables was tested using Pearson's correlation. In case of a significant correlation coefficient $>0.4$ the variable with the strongest relation to appropriate ICD therapy was used for the multivariate model. Variables showing a $p<0.2$ were placed in a multivariate Cox regression model. Echocardiographic outcome parameters were associated with the risk of (first) appropriate ICD therapy using only those events which occurred during follow-up starting \pm 6 
months after echocardiography was performed. KaplanMeier hazard functions were used to determine the association between volume response and reaching an LVEF $>35 \%$ and first appropriate ICD therapy, and significance was assessed by log rank. Subsequently, a multivariate Cox regression model was set up to assess independent association of echocardiographic outcome parameters and the occurrence of appropriate ICD therapy. Both multivariate Cox regression models were performed using backward stepwise regression. Baseline characteristics associated with the outcome measurement predictive of the lowest risk of appropriate ICD therapy were evaluated by performing a backward logistic regression analysis.

\section{Results}

\section{Study population and baseline characteristics}

Detailed baseline characteristics are listed in Table 1 . The final study population consisted of 202 patients. Median age was 67 [14] years, $64 \%(n=129)$ male gender, mean LVEF $21 \pm 6 \%$, and $45 \%(n=91)$ ischaemic cardiomyopathy. Mean QRS duration was $165 \pm 22 \mathrm{~ms}, 59 \%(n=120)$ typical left bundle branch block and $11 \%(n=22)$ paced from the right ventricle.

\section{ICD settings}

Mean heart rate cut-off for the ventricular fibrillation zone was $231 \pm 12$ beats per minute (bpm). In 193 patients also a ventricular tachycardia zone in which ICD therapy was delivered was set at a median of $180 \pm 9 \mathrm{bpm}$.

\section{Primary endpoint}

Total follow-up had a median duration of 37 (IQR 18-46) months in which $25 \%(n=50)$ received appropriate ICD therapy. During the initial 6 months after implant, 8 patients received appropriate ICD therapy of whom 4 had another episode of appropriate ICD therapy during later follow-up. Therefore, a total of 46 (23\%) patients received appropriate ICD therapy after echocardiographic outcome assessment at \pm 6 months. Thirty-nine $(85 \%)$ patients received antitachycardia pacing and $7(15 \%)$ received shock therapy as first appropriate ICD therapy. Eighteen (46\%) patients who received anti-tachycardia pacing as initial appropriate ICD

Table 1 Baseline characteristic comparison based on echocardiographic outcome groups assessed after six months of CRT

\begin{tabular}{|c|c|c|c|c|c|c|}
\hline Variables & $\begin{array}{l}\text { Volume non- } \\
\text { responders }(N=97)\end{array}$ & $\begin{array}{l}\text { Volume responders } \\
(N=105)\end{array}$ & $P$-value & $\begin{array}{l}\mathrm{LVEF} \leq 35 \% \\
(N=151)\end{array}$ & $\begin{array}{l}\text { LVEF > 35\% } \\
(N=51)\end{array}$ & $P$-value \\
\hline$\overline{\text { Age, (median, IQR) yrs }}$ & $67(69-74)$ & $67(60-73)$ & 0.884 & $67(60-73)$ & $65(57-73)$ & 0.659 \\
\hline Males $(\%)$ & $67(69)$ & $62(59)$ & 0.138 & $102(68)$ & $27(53)$ & 0.060 \\
\hline $\operatorname{ICMP}(\%)$ & $55(57)$ & $36(34)$ & 0.001 & $77(51)$ & $14(28)$ & 0.003 \\
\hline NYHA III-IV (\%) & $69(81)$ & $69(76)$ & 0.388 & $111(84)$ & $27(61)$ & 0.002 \\
\hline NYHA I (\%) & $1(1)$ & $1(1)$ & & $0(0)$ & $2(5)$ & \\
\hline NYHA II (\%) & $15(18)$ & $21(23)$ & & $21(16)$ & $15(34)$ & \\
\hline Creatinine (median, IQR) $\mu \mathrm{mol} / \mathrm{L}$ & $114(93-152)$ & $110(83-137)$ & 0.018 & $114(91-144)$ & $104(83-129)$ & 0.048 \\
\hline BNP (median, IQR) pmol/L & $137(71-256)$ & $83(40-194)$ & 0.004 & $132(71-262)$ & 45 (28-99) & $<0.001$ \\
\hline Diuretics (\%) & $88(91)$ & $93(89)$ & 0.759 & $139(93)$ & $45(82)$ & 0.034 \\
\hline Beta-blocker (\%) & $80(83)$ & $86(83)$ & 0.968 & $123(82)$ & $43(84)$ & 0.707 \\
\hline ACE inhibitors (\%) & $69(71)$ & $75(72)$ & 0.877 & $108(72)$ & $36(71)$ & 0.847 \\
\hline $\operatorname{ARB}(\%)$ & $24(25)$ & $28(27)$ & 0.724 & $35(25)$ & $15(29)$ & 0.504 \\
\hline Amiodaron $(\%)$ & $12(12)$ & $12(12)$ & 0.856 & $19(13)$ & $5(10)$ & 0.586 \\
\hline Digoxin $(\%)$ & $24(25)$ & $22(21)$ & 0.545 & $33(22)$ & $13(26)$ & 0.608 \\
\hline Sotalol (\%) & $4(4)$ & $5(5)$ & 0.815 & $6(4)$ & $3(6)$ & 0.574 \\
\hline \multicolumn{7}{|l|}{ Echocardiography } \\
\hline $\operatorname{LVEF}($ mean $\pm \mathrm{SD}) \%$ & $20 \pm 6$ & $21 \pm 6$ & 0.470 & $20 \pm 6$ & $24 \pm 6$ & $<0.001$ \\
\hline LVESV (median, IQR) ml & $182(138-230)$ & $182(136-228)$ & 0.553 & $194(152-239)$ & $136(104-186)$ & $<0.001$ \\
\hline LVEDV (median, IQR) ml & $232(177-278)$ & $224(174-278)$ & 0.514 & $243(196-293)$ & $174(146-229)$ & $<0.001$ \\
\hline $\operatorname{IVMD}($ mean $\pm \mathrm{SD}) \mathrm{ms}(N=189)$ & $36 \pm 23$ & $56 \pm 27$ & $<0.001$ & $41 \pm 27$ & $60 \pm 24$ & $<0.001$ \\
\hline \multicolumn{7}{|l|}{ Electrocardiography } \\
\hline History of AF (\%) & $36(37)$ & $29(28)$ & 0.149 & $45(32)$ & $17(33)$ & 0.838 \\
\hline RV paced $(\%)$ & $12(12)$ & $10(10)$ & 0.516 & $17(11)$ & $5(10)$ & 0.773 \\
\hline $\mathrm{QRSd}(\mathrm{mean} \pm \mathrm{SD}) \mathrm{ms}$ & $161 \pm 22$ & $170 \pm 21$ & 0.004 & $166 \pm 22$ & $165 \pm 22$ & 0.891 \\
\hline LBBB $(\%)$ & $45(46)$ & $75(71)$ & $<0.001$ & $87(58)$ & $33(65)$ & 0.373 \\
\hline
\end{tabular}


Table 2 Uni- and multivariate Cox regression analyses; Baseline characteristics and 6 months echocardiographic outcome measurements associated with appropriate ICD therapy

\begin{tabular}{|c|c|c|c|c|}
\hline \multirow{2}{*}{$\begin{array}{l}\text { Total first events }(N=\mathbf{5 0}) \\
\text { FU start after implant }\end{array}$} & \multicolumn{2}{|l|}{ Univariate } & \multicolumn{2}{|l|}{ Multivariate } \\
\hline & HR $(95 \% \mathrm{CI})$ & $P$-value & HR $(95 \% \mathrm{CI})$ & $P$-value \\
\hline$\overline{\text { Male }}$ & $1.60(0.87-2.95)$ & 0.129 & & \\
\hline ICMP & $1.58(0.90-2.75)$ & 0.109 & $2.00(1.21-3.55)$ & 0.019 \\
\hline Creatinine $>91(\mu \mathrm{mol} / \mathrm{L})$ & $1.75(0.90-3.44)$ & 0.102 & & \\
\hline $\mathrm{BNP}>163(\mathrm{pmol} / \mathrm{L})$ & $3.18(1.81-5.60)$ & $<0.01$ & $3.77(2.10-6.79)$ & $<0.001$ \\
\hline LVEDV $>249 \mathrm{ml}$ & $1.80(1.03-3.13)$ & 0.039 & & \\
\hline \multicolumn{5}{|c|}{$\begin{array}{l}\text { Total (first) events }(N=\mathbf{4 6}) \\
\text { FU start after } 6 \text { months echocardiography }\end{array}$} \\
\hline 6 month-Volume response $(\geq 15 \%)$ & $0.172(0.05-0.55)$ & 0.003 & $0.57(0.30-1.08)$ & 0.086 \\
\hline 6 month-LVEF $>35 \%$ & $0.378(0.20-0.70)$ & 0.002 & $0.23(0.07-0.80)$ & 0.020 \\
\hline
\end{tabular}

$B N P$ B-type natriuretic peptide, $C I$ confidence interval, $F U$ follow up, $H R$ hazard ratio, ICMP ischemic cardiomyopathy, $L V E D V$ left ventricular end diastolic volume, $L V E F$ left ventricular ejection fraction.
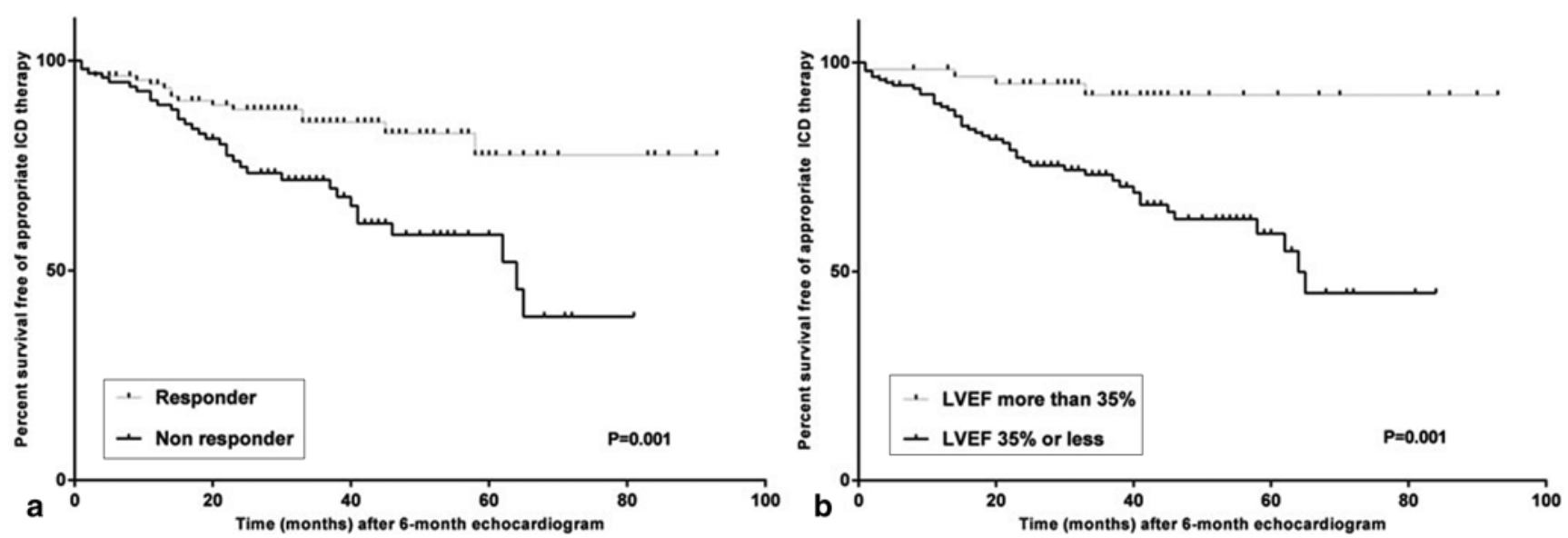

Fig. 1 Kaplan-Meier plots of percent survival free of appropriate ICD therapy after 6-month echocardiogram by (a) volume response and (b) $\mathrm{LVEF} \leq>35 \%$

therapy received appropriate shock therapy after unsuccessful pacing or later during follow-up.

\section{Baseline predictors of first appropriate ICD therapy}

Univariable and multivariable hazard ratios (HR) for the occurrence of appropriate ICD therapy for baseline characteristics are listed in Table 2. BNP $>163 \mathrm{pmol} / 1$ and ischaemic cardiomyopathy were independently associated with a significantly higher risk of ventricular arrhythmias, HR 3.8 $p<0.001$ and HR 2.0, $p=0.019$, respectively. Four $(7 \%)$ out of 61 patients with both a BNP $\leq 163 \mathrm{ml}$ at baseline and nonischaemic cardiomyopathy received appropriate ICD therapy.

\section{Echocardiographic outcome and first appropriate ICD therapy}

After 6 months of CRT, 105 (52\%) patients showed volume response. Fifteen (14\%) responders received appropriate ICD therapy compared with $31(32 \%)$ non-responders $(p=0.001)$ (Fig. 1a). Fifty-one $(25 \%)$ patients reached an
LVEF $>35 \%$ after 6 months of which three $(6 \%)$ received appropriate ICD therapy compared with 43 patients (29\%) with an LVEF $\leq 35 \%$ ( $p=0.001$, Fig. $1 b)$. Four ( $4 \%$ ) patients who were defined as volume responders at 6 months received appropriate ICD therapy prior to this echocardiogram. None of the patients with an LVEF $>35 \%$ after 6 months received appropriate ICD therapy during the initial 6 months.

Of the volume responders, $44 \%$ reached an LVEF $>35 \%$ while $90 \%$ of patients with an LVEF $>35 \%$ after 6 months were also defined as volume responders $(\geq 15 \%)$. Multivariate analysis of 6-month echocardiographic outcome variables showed only LVEF $>35 \%$ to be significantly associated with a lower risk of ventricular arrhythmias (HR $0.23,95 \%$ CI $0.07-0.78, p=0.020$ ) (Table 2).

Factors independently associated with an LVEF $>35 \%$ after 6 months of CRT were non-ischaemic cardiomyopathy (OR 2.6 95\% CI 1.12-5.97, $p=0.026$ ), a higher baseline LVEF (OR 1.16, $95 \%$ CI 1.08-1.24, $P<0.001$ ) and a longer IVMD (OR 1.03, $95 \%$ CI 1.01-1.04, $p<0.001$ ) (Table 3 ). 
Table 3 Multivariate logistic regression model; factors associated with LVEF $>35 \%$ at 6 months

Factors associated with LVEF $>35 \%$ after 6 months of CRT

\begin{tabular}{llll}
\hline & OR & $95 \%$ CI & $P$-value \\
\hline NICMP & 2.590 & $1.123-5.972$ & 0.026 \\
LVEF pre CRT $(\%)$ & 1.158 & $1.084-1.237$ & $<0.001$ \\
IVMD pre CRT $(\mathrm{ms})$ & 1.025 & $1.009-1.042$ & $<0.001$
\end{tabular}

Logistic regression model, corrected for gender and diuretic use.

$B N P$ B-type natriuretic peptide, $C I$ coincidence interval, IVMD interventricular mechanical delay, $L V E F$ left ventricular ejection fraction, NICMP non-ischemic cardiomyopathy.

\section{Sub-analysis aetiology of heart failure}

Twenty-seven $(30 \%)$ patients with ischaemic cardiomyopathy received appropriate ICD therapy compared with $23(21 \%)$ with non-ischaemic cardiomyopathy $(p=0.105)$. Thirty-six (40\%) patients with ischaemic cardiomyopathy showed volume response and only $14(15 \%)$ reached an LVEF $>35 \%$. Eight $(22 \%)$ ischaemic volume responders received appropriate ICD therapy compared with 19 (35\%) non-responders $(p=0.301)$. For those ischaemic patients with an LVEF $>35 \%$ after 6 months of CRT, there was a trend towards a lower risk of appropriate ICD therapy $(p=0.062)$.

\section{Inappropriate ICD therapy and mortality}

A total of $21(10.4 \%)$ patients received inappropriate ICD therapy during follow-up. Ten (5\%) patients received an inappropriate shock including two responders: one of these two patients reached an LVEF $>35 \%$. During follow-up 39 (19.3\%) patients died: 27 (27.8\%) non-responders, and 12 $(11.4 \%)$ responders $(p=0.002)$. No patients with an LVEF $>35 \%$ died during follow-up. Annual mortality rates were $10.2 \%$ in non-responders, $3.4 \%$ in responders, $8.3 \%$ in patients with an LVEF $\leq 35 \%$ and $0 \%$ in patients with an LVEF $>35 \%$ after 6 months of CRT.

\section{Discussion}

In the current study we showed that ischaemic cardiomyopathy and a BNP level $>163 \mathrm{pmol} / \mathrm{l}$ at baseline were associated with an increased risk of ventricular arrhythmias after CRT implantation. Other studies evaluating baseline predictors of ventricular arrhythmias in a CRT population found variable results but identified gender, no beta-blocker or ACE inhibitor use, severely decreased LVEF and NYHA class IV as predictors of ventricular arrhythmias [18-20]. Friedman et al. found that LV end-diastolic diameter was a strong predictor of ventricular arrhythmias in a CRT population even when corrected for reverse remodelling after CRT
[20]. Moreover, Van der Heijden et al. found LV end-systolic volume $>130 \mathrm{ml}$ predictive of appropriate ICD therapy [21]. Although we could not confirm these results we did see a trend for larger cardiac volumes at baseline to be associated with a higher risk of appropriate ICD therapy. Furthermore, most of these baseline predictors of ventricular arrhythmias after CRT implantation seem to relate to the degree of heart failure prior to implant, as does BNP level, which was a predictor in our population. The variability among studies indicates that prior to implant it is hard to identify valid predictors of ventricular arrhythmias, probably caused by the dynamic nature of CRT which induces changes in baseline variables associated with processes important in arrhythmogenesis, such as wall stress [4], oxygen consumption and supply [22], and neurohormonal activation [23].

\section{CRT and arrhythmogenesis}

Regarding our second aim, we found that patients with a favourable echocardiographic outcome at 6 months had a significantly lower risk of ventricular arrhythmias compared with those showing a less favourable outcome. Although the majority of previous single-centre studies analysing the effect of reverse remodelling after CRT implantation on the risk of ventricular arrhythmias found comparable results, there was some controversy. The observed discrepancy might be due to different definitions of volume response, inclusion of both primary and secondary prophylactic ICD patients and different follow-up durations [2-6, 19]. We showed absolute LVEF at 6 months to be a stronger predictor of ventricular arrhythmias than the occurrence of volume response. An explanation might be that volume response only reflects a relative improvement while absolute LVEF after CRT implantation is more strongly associated with pre- and post-CRT cardiac volumes. Sub-analysis of heart failure aetiology showed that volume response in patients with ischaemic cardiomyopathy was not significantly associated with the risk of ventricular arrhythmias while there was a trend towards a significant correlation with LVEF at 6 months and appropriate ICD therapy. These findings might have multiple explanations. First of all, it is known that patients with ischaemic cardiomyopathy respond less to CRT than those with non-ischaemic cardiomyopathy [24]. Moreover, the mechanism of ventricular arrhythmias in ischaemic cardiomyopathy patients is most often a re-entry tachyarrhythmia involving the scar tissue [25]. It is unlikely that response to CRT is of influence on these ventricular arrhythmias. Finally, response to CRT enables the patient to perform at a higher level of activity; in patients with ischaemic cardiomyopathy this could increase the ischaemic events and therefore trigger ischaemia-induced ventricular arrhythmias. 
Although the majority of the evidence shows that favourable echocardiographic outcome after CRT is associated with a lower risk of ventricular arrhythmias, this is compared with patients who show a less favourable response to CRT. It is unclear whether CRT itself is antiarrhythmic as most studies did not have an ICD-only group for comparison. Moreover, there are also some suggestions for a proarrhythmic effect based on small case cohorts and reports [26]. Most of these proarrhythmic events after CRT are linked to pacing in scar tissue but it is also possible that reversal of transmural activation pattern, due to LV epicardial pacing, increases repolarisation dispersion and thereby increases the risk of ventricular arrhythmias. Although we did not have an ICD-only group either, previous occurrence of first appropriate ICD therapy among primary prophylactic one- or two-chamber ICD patients implanted in our hospital was analysed by Wijers et al. [27]. Eighteen percent $(N=55)$ (mean LVEF $24 \pm 6 \%)$ received appropriate ICD therapy during a follow-up of $31 \pm 17$ months. As $25 \%$ of our CRT-D patients received appropriate ICD therapy during a median follow-up of 37 months, it seems the overall effect of CRT on arrhythmogenesis is neutral. However, when looking at the occurrence of ventricular arrhythmias in non-responders $(32 \%)$ and responders $(14 \%)$ it suggests competing proarrhythmic effects in non-responders and antiarrhythmic effects in responders. Although this is in line with the results of the REVERSE study and the MADIT CRT trial, any conclusion based on comparison of these numbers is limited since an adequate baseline comparison was not performed and therefore other patient characteristics could have influenced the results $[2,3]$.

\section{CRT-D or CRT-P}

The majority of CRT devices implanted in the Netherlands are CRT-D devices [28]. Even though all patients eligible for CRT have an indication for primary prophylactic ICD therapy based on their depressed LVEF, the survival benefit of CRT-D over CRT-P is still matter of debate. In the choice between CRT-D and CRT-P, the 2013 ESC guideline, endorsed by our national society, the NVVC, states that no strict recommendations on device choice can be made due to insufficient evidence from randomised controlled trials. The guideline merely aims to offer guidance regarding the selection of patients for CRT-D versus CRT-P based on clinical condition, device-related complications as inappropriate ICD therapy, and costs [1]. We showed that $10 \%$ of our CRT-D population received inappropriate ICD therapy, which is associated with healthcare costs, and CRT-D device costs are much higher than CRT-P costs. Therefore, careful consideration in the choice of CRT device is warranted. Moreover, basing CRT device choice on pre-CRT clinical condition is questionable as we, and the majority of studies, have shown response to CRT to influence the risk of ventricular arrhythmias.

Only first appropriate ICD therapy was analysed, as we opted that the risk of first appropriate ICD therapy was of most interest in the decision to implant a CRT-D or a CRT-P, because one episode of appropriate therapy can in theory have prevented a sudden cardiac death. Whether the patient receives more appropriate ICD therapy after this episode is of limited additional value in CRT device choice.

Patients with an LVEF $>35 \%$ after CRT implantation were at a low risk $(6 \%)$ of appropriate ICD therapy during 37 months of follow-up. Previously, Van Boven et al. [8] actually showed that none of the patients who reached an LVEF $\geq 35 \%$ after 4 months of CRT needed appropriate shock therapy during a follow-up of 3 years. Manfredi et al. [9] and Schaer et al. [10], who used a higher cut-off in LVEF, found similar results and Ruwald et al. [11] showed with 752 patients from the MADIT CRT trial that patients who achieve LVEF normalisation ( $>50 \%$ ) have a very low absolute and relative risk of ventricular tachyarrhythmias within 2.2 years of follow-up.

Therefore it would be of great value to correctly predict which patients are most likely to reach an LVEF $>35 \%$ as these patients could be candidates for CRT-P implantation. We propose that the characteristics associated with reaching this LVEF in our cohort, namely non-ischaemic cardiomyopathy, higher IVMD, and higher LVEF, could be used as pre-implantation predictors of patients suitable for CRT-P.

Although these characteristics have also been previously linked to response to CRT in other cohorts [29, 30], prediction of echocardiographic outcome has proven to be difficult. As non-responders might actually be at increased risk of ventricular arrhythmias and responders could still be at risk during the initial 6 months the association found might be of more value for the decision to downgrade a CRT-D in case of generator replacement. Meaning that if a patient has an LVEF $>35 \%$ at the time of generator replacement, CRT-D downgrading to a CRT-P could be considered. This consideration should be restricted to patients with non-ischaemic cardiomyopathy as the association between echocardiographic outcome and appropriate ICD therapy was much weaker in ischaemic cardiomyopathy patients. Moreover, it is unclear whether the relationship between LVEF and risk of ventricular arrhythmias at the time of generator replacement, approximately 5 years after implant, is comparable with the relationship found during the currently evaluated period. We recommend further research into this correlation.

\section{Limitations}

A limitation of this retrospective analysis is the preselected population of CRT patients based on the need for paired 
echocardiography data. For any recommendation on CRT device choice an important limitation is the relatively short follow-up as we do not know what happens to the risk of ventricular arrhythmias after the median 37 months of our follow-up. Some minor limitations are due to the retrospective design and reflect the incomplete data concerning NYHA class and BNP.

\section{Conclusion}

Baseline prediction of the risk of ventricular arrhythmias in patients eligible for CRT remains challenging. After 6 months of CRT, absolute LVEF is the strongest predictor of ventricular arrhythmias. Patients with an LVEF $>35 \%$ at 6 months are at a low risk of appropriate ICD therapy. Therefore LVEF post-CRT could be used to decide on downgrading of a CRT-D to a CRT-P in case of generator replacement.

\section{Funding None.}

\section{Conflict of interests None declared}

Open Access This article is distributed under the terms of the Creative Commons Attribution 4.0 International License (http://creativecommons.org/licenses/by/4.0/), which permits use, duplication, adaptation, distribution, and reproduction in any medium or format, as long as you give appropriate credit to the original author(s) and the source, provide a link to the Creative Commons license, and indicate if changes were made.

\section{References}

1. Brignole M, Auricchio A, Baron-Esquivias G, et al. 2013 ESC Guidelines on cardiac pacing and cardiac resynchronization therapy. Rev Esp Cardiol. 2014;67:58.

2. Gold MR, Linde C, Abraham WT, Gardiwal A, Daubert JC. The impact of cardiac resynchronization therapy on the incidence of ventricular arrhythmias in mild heart failure. Hear Rhythm. 2011;8:679-84.

3. Barsheshet A, Wang PJ, Moss AJ, et al. Reverse remodeling and the risk of ventricular tachyarrhythmias in the MADIT-CRT (Multicenter Automatic Defibrillator Implantation Trial-Cardiac Resynchronization Therapy). J Am Coll Cardiol. 2011;57:2416-23.

4. Di Biase L, Gasparini M, Lunati M, et al. Antiarrhythmic effect of reverse ventricular remodeling induced by cardiac resynchronization therapy. the InSync ICD (Implantable Cardioverter-Defibrillator) Italian Registry. J Am Coll Cardiol. 2008;52:1442-9.

5. Shahrzad S, Soleiman NK, Taban S, et al. The effect of Left Ventricular (LV) remodeling on ventricular arrhythmia in cardiac resynchronization therapy (CRT-D) patients (Antiarrhythmic Effect of CRT). Pacing Clin Electrophysiol. 2012;35:592-7.

6. Steffel J, Milosevic G, Hurlimann A, et al. Characteristics and long-term outcome of echocardiographic super-responders to cardiac resynchronisation therapy: "real world" experience from a single tertiary care centre. Heart. 2011;97:1668-74.
7. Frigerio M, Lunati M, Pasqualucci D, et al. Left ventricular ejection fraction overcrossing $35 \%$ after one year of cardiac resynchronization therapy predicts long term survival and freedom from sudden cardiac death: single center observational experience. Int J Cardiol. 2014;17:64-71.

8. Van Boven N, Bogaard K, Ruiter J, et al. Functional response to cardiac resynchronization therapy is associated with improved clinical outcome and absence of appropriate shocks. J Cardiovasc Electrophysiol. 2013;24:316-22.

9. Manfredi JA, Al-Khatib SM, Shaw LK, et al. Association between left ventricular ejection fraction post-cardiac resynchronization treatment and subsequent implantable cardioverter defibrillator therapy for sustained ventricular tachyarrhythmias. Circ Arrhythmia Electrophysiol. 2013;6:257-64.

10. Schaer BA, Osswald S, Di Valentino M, et al. Close connection between improvement in left ventricular function by cardiac resynchronization therapy and the incidence of arrhythmias in cardiac resynchronization therapy-defibrillator patients. Eur J Heart Fail. 2010;12:1325-32.

11. Ruwald MH, Solomon SD, Foster E, et al. Left ventricular ejection fraction normalization in cardiac resynchronization therapy and risk of ventricular arrhythmias and clinical outcomes: results from the Multicenter Automatic Defibrillator Implantation Trial With Cardiac Resynchronization Therapy. Circulation. 2014;130:2278-86.

12. Van der Wall EE. Improvement in CRT: new strategies, better choices. Neth Heart J. 2014;22:413-4.

13. Brignole M, Auricchio A, Baron-Esquivias G, et al. 2013 ESC guidelines on cardiac pacing and cardiac resynchronization therapy: the task force on cardiac pacing and resynchronization therapy of the European Society of Cardiology (ESC). Developed in collaboration with the European Heart Rhythm Association. Europace. 2013;15:1070-118.

14. Moss AJ, Hall WJ, Cannom DS, et al. Cardiac-resynchronization therapy for the prevention of heart-failure events. N Engl J Med. 2009;361:1329-38.

15. Surawicz B, Childers R, Deal BJ, et al. AHA/ACCF/HRS recommendations for the standardization and interpretation of the electrocardiogram: part III: intraventricular conduction disturbances: a scientific statement from the American Heart Association Electrocardiography and Arrhythmias Committee. J Am Coll Cardiol. 2009;53:976-81.

16. McMurray JJV, Adamopoulos S, Anker SD, et al. ESC Guidelines for the diagnosis and treatment of acute and chronic heart failure 2012: the Task Force for the Diagnosis and Treatment of Acute and Chronic Heart Failure 2012 of the European Society of Cardiology. Developed in collaboration with the Heart. Eur Heart J. 2012;33:1787-847.

17. Chung ES, Leon AR, Tavazzi L, et al. Results of the Predictors of Response to CRT (PROSPECT) trial. Circulation. 2008;117:2608-16.

18. Desai AD, Burke MC, Hong TE, et al. Predictors of appropriate defibrillator therapy among patients with an implantable defibrillator that delivers cardiac resynchronization therapy. J Cardiovasc Electrophysiol. 2006;17:486-90.

19. Soliman O II, Theuns DAMJ, Dalen BM van, et al. Prediction of appropriate defibrillator therapy in heart failure patients treated with cardiac resynchronization therapy. Am J Cardiol. 2010;105:105-11.

20. Friedman DJ, Altman RK, Orencole M, et al. Predictors of sustained ventricular arrhythmias in cardiac resynchronization therapy. Circ Arrhythmia Electrophysiol. 2012;5:762-72. 
21. Van der Heijden AC, Höke U, Thijssen J, et al. Super-responders to cardiac resynchronization therapy remain at risk for ventricular arrhythmias and benefit from defibrillator treatment. Eur J Heart Fail. 2014;16:1104-11.

22. Linde C, Leclercq C, Rex S, et al. Long-term benefits of biventricular pacing in congestive heart failure: results from the MUltisite STimulation In Cardiomyopathy (MUSTIC) study. J Am Coll Cardiol. 2002;40:111-8.

23. Braun MU, Rauwolf T, Zerm T, Schulze M, Schnabel A, Strasser RH. Long term biventricular resynchronisation therapy in advanced heart failure: effect on neurohormones. Heart. 2005;91:601-5.

24. Chen Y, Duan C, Liu F, Shen S, Chen P, Bin J. Impact of etiology on the outcomes in heart failure patients treated with cardiac resynchronization therapy: a meta-analysis. PLoS One. 2014;9:e94614.

25. Hynes BJ, Luck JC, Wolbrette DL, Boehmer J, Naccarelli GV. Arrhythmias in patients with heart failure. Curr Treat Options Cardiovasc Med. 2002;4:467-85.

26. Shukla G, Chaudhry GM, Orlov M, Hoffmeister P, Haffajee C. Potential proarrhythmic effect of biventricular pacing: fact or myth? Heart Rhythm. 2005;2:951-6.
27. Wijers SC, Kolk BYM van der, Tuinenburg AE, Doevendans PAF, Vos MA, Meine M. Implementation of guidelines for implantable cardioverter-defibrillator therapy in clinical practice: which patients do benefit? Neth Heart J. 2013;21:274-83.

28. Raatikainen MJP, Arnar DO, Zeppenfeld K, Merino JL, Kuck $\mathrm{K}-\mathrm{H}$, Hindricks G. Current trends in the use of cardiac implantable electronic devices and interventional electrophysiological procedures in the European Society of Cardiology member countries: 2015 report from the European Heart Rhythm Association. Europace. 2015; 17:iv1-72.

29. Goldenberg I, Moss AJ, Hall WJ, et al. Predictors of response to cardiac resynchronization therapy in the Multicenter Automatic Defibrillator Implantation Trial with Cardiac Resynchronization Therapy (MADIT-CRT). Circulation. 2011;124:1527-36.

30. Van Bommel RJ, Bax JJ, Abraham WT, et al. Characteristics of heart failure patients associated with good and poor response to cardiac resynchronization therapy: a PROSPECT (Predictors of Response to CRT) sub-analysis. Eur Heart J. 2009;30:2470-7. 